\title{
Stationary Configuration of some Optimal Shaping
}

\author{
Giuseppe Buttazzo and Al-hassem Nayam
}

\begin{abstract}
We consider the problem of optimal location of a Dirichlet region in a $d$-dimensional domain $\Omega$ subjected to a given right-hand side $f$ in order to minimize some given functional of the configuration. While in the literature the Dirichlet region is usually taken $d-1$ dimensional, in this shape optimization problems, we consider two classes of control variables, namely the class of one dimensional closed connected sets of finite one dimensional Hausdorff measure and the class of sets of points of finite cardinality, and we give a necessary condition of optimality.
\end{abstract}

Keywords: shape optimization, p-Laplacian, higher codimension, necessary conditions of optimality.

Biographical notes: Giuseppe Buttazzo graduated in Mathematics at the University of Pisa in 1976, and received in the same year the Diploma of Scuola Normale Superiore di Pisa, where he won a national competition as a student. He made the Ph. D. studies from 1976 until 1980 at Scuola Normale di Pisa, where he also got his first permanent position as a researcher in 1980. He obtained the full professorship in Mathematical Analysis at University of Ferrara in 1986, and moved in 1990 to University of Pisa, where he presently works at the Department of Mathematics. His main research interests include calculus of variations, partial differential equations, optimization problems, optimal control theory. On these subjects he wrote more than 150 scientific papers and several books.

Al-hassem Nayam received his PhD Degree in mathematics at University of Pisa in 2011. His PhD Thesis mainly deals with the shape optimization problems of higher codimension. His research interest lies on asymptotic shapes, necessary condition of optimality and regularity of optimal shapes. At present he works at International Centre for Theoretical Physics (ICTP) in Trieste, Italy. 


\section{Introduction}

We consider the problem of finding the optimal location of a Dirichlet region $\Sigma$ in a $d$-dimensional domain $\Omega$ associated the $p$-Laplacian

$$
\begin{cases}-\operatorname{div}\left(|\nabla u|^{p-2} \nabla u\right)=f & \text { in } \Omega \backslash \Sigma \\ u=0 & \text { on } \partial \Omega \cup \Sigma\end{cases}
$$

where the right hand side $f$ is given as a nonnegative element of $L^{p^{\prime}}(\Omega)$, being $p^{\prime}$ the conjugate exponent of $p$. The functional $\mathcal{F}$ we consider as a cost is defined by

$$
\mathcal{F}(\Sigma)=\int_{\Omega} F(x, u(x), \nabla u(x)) d x
$$

where $u$ is the unique solution of equation (1) and $F: \Omega \times \mathbb{R} \times \mathbb{R}^{d} \rightarrow \mathbb{R}$ is a Carathéodory function. The shape optimization problems we consider consists in the minimization of the functional $\mathcal{F}$ over two classes of admissible control variables $\Sigma$. The first class consists of all closed connected subsets $\Sigma$ of $\Omega$ whose one-dimensional Hausdorff measure $\mathcal{H}^{1}(\Sigma)$ (sometime called the total length of $\Sigma$ ) is uniformly bounded by some constant $L$, while the second is the class of discrete subsets of $\Omega$ whose 0 -dimensional Hausdorff measure (i.e. their cardinality) does not exceed a given number $n$.

The existence of an optimal configuration for the two optimization problems described above can be obtained by using a generalization of the Šverák's compactness result (see [11] for $p=2$ and [3] for a general $p$ ). In this paper, we consider a penalized version of these shape optimization problems (see (2) and (3) ) by adding to the cost functional $\mathcal{F}$ a Lagrange multiplier penalization of the form $\lambda \mathcal{H}^{1}(\Sigma)$ and $\lambda \mathcal{H}^{0}(\Sigma)$ respectively. This problem has been considered in [4] in the simplest case when $p=2$, where the PDE (11) reduces to the classical Laplace equation. Moreover, it has been shown (see [5]) that, for the particular case where $F(x, u, \xi)=f(x) u$, the limit problem, as $p \rightarrow+\infty$, is the minimum problem for the average distance functional

$$
\mathcal{F}(\Sigma)=\int_{\Omega} \operatorname{dist}(x, \Sigma \cup \partial \Omega) f(x) d x,
$$

where $\operatorname{dist}(x, E)$ denotes the distance between the point $x$ and the set $E$.

For the well posedness of the minimum problems (21) and (3) the constraint $p>d-d_{\Sigma}$, where $d_{\Sigma}$ is the dimension of $\Sigma$, has to be imposed in order to have Dirichlet regions of positive $p$-capacity. Let us mention that the regularity of the optimal sets (in the case of problem (2) ) is still an open problem, even in the simplest two dimensional setting with $p=2$ and $F=f(x) u$. 


\section{Setting of the problem and existence of mini- mizers}

We consider the following classes of control variables:

$$
\begin{aligned}
\mathcal{A}(\Omega) & :=\left\{\Sigma \subset \Omega: \Sigma \text { closed connected, } \mathcal{H}^{1}(\Sigma)<+\infty\right\} \\
\mathcal{B}(\Omega): & =\left\{\Sigma \subset \Omega: \Sigma \text { discrete, } \mathcal{H}^{0}(\Sigma)=\#(\Sigma)<+\infty\right\}
\end{aligned}
$$

and the shape optimization problems

$$
\begin{aligned}
& \min \left\{\mathcal{F}(\Sigma)+\lambda \mathcal{H}^{1}(\Sigma): \Sigma \in \mathcal{A}(\Omega)\right\} \\
& \min \left\{\mathcal{F}(\Sigma)+\lambda \mathcal{H}^{0}(\Sigma): \Sigma \in \mathcal{B}(\Omega)\right\} .
\end{aligned}
$$

The penalization terms $\lambda \mathcal{H}^{1}(\Sigma)$ and $\lambda \mathcal{H}^{0}(\Sigma)$ with $\lambda>0$ replace the constraint on $\mathcal{H}^{1}(\Sigma)$ and $\mathcal{H}^{0}(\Sigma)$ and prevent the minimizing sequences to spread over all the domain $\Omega$ and hence getting a trivial solution. The existence of minimizers in the two shape optimization problems (2) and (3) is a consequence of the Šverák continuity-compactness result (see [11] for $p=2$ and [3] for general $p$ ) and the Blaschke and Gołab theorems. For the convenience of the reader let us give some details on the existence of an optimal shape. Let $\left\{\Sigma_{n}\right\}_{n} \subset \mathcal{A}(\Omega)$ be a minimizing sequence in the optimization problem (2), then there exists a constant $C>0$ such that $\sup _{n}\left\{\mathcal{F}\left(\Sigma_{n}\right)+\mathcal{H}^{1}\left(\Sigma_{n}\right)\right\} \leq C$. Since $\left\{\Sigma_{n}\right\}_{n}$ is a sequence a closed connected subsets of $\Omega$ such that $\sup _{n} \mathcal{H}^{1}\left(\Sigma_{n}\right) \leq C$, by Blaschke theorem (compactness of the sequence $\left\{\Sigma_{n}\right\}_{n}$ in the Hausdorff topology) and by Gołab theorem (lower semicontinuity of the $\mathcal{H}^{1}$ with respect to the Hausdorff topology), up to extracting a subsequence, $\left\{\Sigma_{n}\right\}_{n}$ converges in Hausdorff distance to some $\Sigma \in \mathcal{A}(\Omega)$ and $\mathcal{H}^{1}(\Sigma) \leq \liminf \operatorname{in}_{n \rightarrow \infty} \mathcal{H}^{1}\left(\Sigma_{n}\right)$. For the lower semicontinuity of the energy part of the functional we need the Sverák continuity-compactness result which is stated in this terms: let $\left\{\Omega_{n}\right\}_{n}$ be a sequence of open and bounded sets contained in a fix bounded set $D$. If we assume that the number of the connected components of $D \backslash \Omega_{n}$ is uniformly bounded by some number $k$, then $\left\{\Omega_{n}\right\}_{n}$ converges in the Hausdorff topology to some open and bounded set $\Omega \subset D$ and the number of the connected components of $D \backslash \Omega$ is less or equal to $k$. Moreover, if we denote by $u_{n} \in W_{0}^{1, p}\left(\Omega_{n}\right)$ the distributional solution of the $p$-Laplace equation

$$
\left\{\begin{array}{l}
-\Delta_{p} u_{n}=f \quad \text { in } \Omega_{n} \\
u_{n} \in W_{0}^{1, p}\left(\Omega_{n}\right)
\end{array}\right.
$$

for some $f$ in $W^{-1, p^{\prime}}(D)$, then up to subsequence, $u_{n}$ converges strongly in $W^{1, p}(D)\left(u_{n}\right.$ are extended by zero outside $\left.\Omega_{n}\right)$ to the function $u$ which is the distributional solution of the equation

$$
\left\{\begin{array}{l}
-\Delta_{p} u=f \quad \text { in } \Omega \\
u \in W_{0}^{1, p}(\Omega) .
\end{array}\right.
$$


This result is interesting only in the case where $p$ satisfies $d-1<p \leq d$ because the case where $p>d$ is trivial due to the fact that functions in $W^{1, p}(D)$ are continuous and the convergence of solutions follows easily. To apply this result to our problem, we choose $\Omega_{n}=D \backslash \Sigma_{n}$ and notice that $\left\{\Omega_{n}\right\}_{n}$ converges to $\Omega=D \backslash \Sigma$ in the Hausdorff topology where $\Sigma$ is the limit of $\Sigma_{n}$. From the hypothesis of the function $F$ and the continuity with respect to the domains variation of solutions, the lower semicontinuity follows easily and also the existence of an optimal shape. The existence of an optimal set in the problem (2) is even easier due to the fact that $p>d$.

Our goal is to derive first order necessary conditions of optimality, assuming that the solutions $\Sigma$ of the minimum problems are regular as necessary. Notice that, since every set in $\Sigma \in \mathcal{A}(\Omega)$ is countably $\mathcal{H}^{1}$ rectifiable, it can be written as an union of countable $C^{1}$ curves and a set of $\mathcal{H}^{1}$ measure zero.

We distinguish three cases according to technical computations. We consider the following classes of control variables $\Sigma$ : the class of closed connected subsets of $\mathbb{R}^{2}$, the class of points of $\mathbb{R}^{d}$ for $d>1$, and the class of closed connected subsets of $\mathbb{R}^{d}$ for $d>2$. In the two last cases, some extra difficulties occur because of the co-dimension of $\Sigma$ which is greater than 1 . For simplicity, we assume that $\Omega$ has a smooth boundary and $u=0$ on $\partial \Omega \cup \Sigma$; we also assume as much as needed the regularity on the data. Before looking for the necessary conditions of optimality, we recall some definitions and results which will be helpful; we refer to [2] for the details.

For a measure $\mu$ we denote for $\mu$ a.e. $x$ by $P_{\mu}(x, \cdot): \mathbb{R}^{d} \rightarrow \operatorname{Tan}(\mu, x)$ the orthogonal projection of $\mathbb{R}^{d}$ on $\operatorname{Tan}(\mu, x)$ where $\operatorname{Tan}(\mu, x)$ stands for the tangent space of $\mu$ at $x$ that is the set of all tangent measures to $\mu$ at the point $x$ (see for instance [1]).

Definition 1 The curvature of $\mu$ is defined as the vector valued distribution

$$
H_{\mu}:=\operatorname{div}\left(P_{\mu} \mu\right) .
$$

In other words $H_{\mu}$ is defined by

$$
\left.\left\langle H_{\mu}, X\right\rangle=-\int_{\mathbb{R}^{d}} \operatorname{div}^{\mu} X d \mu \quad \forall X \in C_{c}^{\infty}\left(\mathbb{R}^{d}\right), \mathbb{R}^{d}\right),
$$

where $\operatorname{div}^{\mu} X=\sum_{j=1}^{d}\left(P_{\mu}\left(\nabla X^{j}\right)\right)_{j}$.

We denote by $\mathcal{M}_{B C}$ the set of all positive and finite Borel regular measures of $\mathbb{R}^{d}$ whose curvature is a Borel regular measure with finite total mass. Since the curvature $H_{\mu}$ of a measure $\mu \in \mathcal{M}_{B C}$ is not necessary absolutely continuous with respect to $\mu$, by Radon-Nikodym theorem, we can write

$$
H_{\mu}=h(\mu) \mu+\partial \mu
$$


where $h(\mu) \in L_{\mu}^{1}\left(\mathbb{R}^{d}, \mathbb{R}^{d}\right)$ is the density of $H_{\mu}$ with respect to $\mu$ (also called the pointwise curvature) and $\partial \mu$ is the singular part of $H_{\mu}$ with respect to $\mu$ (also called the boundary of $\mu$ ).

If $\mu=\mathcal{H}^{k}\left\llcorner\Sigma\right.$, with $\Sigma$ a $C^{2} k$-manifold with boundary in $\mathbb{R}^{d}$, then by classical divergence theorem we have

$$
H_{\mu}=\nu \mathcal{H}^{k-1}\left\llcorner\partial \Sigma+h \mathcal{H}^{k}\llcorner\Sigma,\right.
$$

where $h$ stands for the mean curvature vector of $\Sigma$ and $\nu$ the co-normal unit vector of $\partial \Sigma$. When the tangent space to $\mu$ is reduced to zero $\mu$ a.e., $H_{\mu}$ is zero. This is for instance the case where $\mu$ is a finite sum of Dirac masses, or $\mu$ is concentrated on an $\alpha$-dimensional Cantor subset $C$ of $[0,1]$ with $0<\alpha<1$ and $\mathcal{H}^{\alpha}(C) \in(0,+\infty)$.

Definition 2 Let $\Sigma$ be a countably $\mathcal{H}^{k}$ rectifiable set and $\mu=\theta \mathcal{H}^{k}\llcorner\Sigma$ be the associated rectifiable measure. A function $h \in L_{\mu}^{1}\left(\Sigma, \mathbb{R}^{d}\right)$ is said to be the generalized mean curvature of $\Sigma$ if

$$
\int_{\mathbb{R}^{d}} \operatorname{div}^{\Sigma} X d \mu=-\int_{\mathbb{R}^{d}} X \cdot h d \mu \quad \forall X \in C_{c}^{\infty}\left(\mathbb{R}^{d}, \mathbb{R}^{d}\right) .
$$

In this case we denote the generalized mean curvature of $\Sigma$ by $H_{\Sigma}$.

Theorem 3 Let $\left(\mu_{r}\right)_{r}$ be a bounded sequence in $\mathcal{M}_{B C}$ weakly converging to some measure $\mu$ and assume that $\operatorname{dim} \operatorname{Tan}\left(\mu_{r}\right) \mu_{r}$ weakly converges to g $\mu$. Then the condition

$$
\operatorname{dim} \operatorname{Tan}(\mu, x) \leq g(x) \quad \mu-\text { a.e. }
$$

is necessary and sufficient to have

$$
P_{\mu_{r}} \mu_{r} \rightarrow P_{\mu} \mu .
$$

In this case we have

$$
H_{\mu_{r}} \rightarrow H_{\mu}
$$

Proof: see [2]

Let $\Omega$ be an open subset of $\mathbb{R}^{d}$ and $F: \Omega \times \mathbb{R} \times \mathbb{R}^{d} \rightarrow[0,+\infty]$ be a positive Carathéodory function. We assume $F$ smooth and satisfying the growth condition

$$
F(x, u, z) \leq a(x)+|u|^{p}+|z|^{p},
$$

where $a$ is an $L^{1}(\Omega)$ function. We consider the functional

$$
\mathcal{F}(\Sigma):=\int_{\Omega \backslash \Sigma} F(x, u(x), \nabla u(x)) d x+\lambda m(\Sigma)
$$

where $m(\Sigma)$ is either $\mathcal{H}^{1}(\Sigma)$ if $\Sigma$ is a closed connected one dimensional set or $\mathcal{H}^{0}(\Sigma)$ if $\Sigma$ is a discrete set of points and $u$ the solution of the equation (1). We are interested in the necessary conditions of optimality satisfied by the minimizers of $\mathcal{F}$. From now on we assume optimal sets in the case of closed connected sets to be of class $C^{1, \alpha}$ for some $0<\alpha \leq 1$ that is locally graph of $C^{1, \alpha}$ functions. 


\section{Case of closed connected subsets in $\mathbb{R}^{2}$}

Let $u$ be the weak solution of the state equation

$$
\begin{cases}-\operatorname{div}\left(|\nabla u|^{p-2} \nabla u\right)=f & \text { in } \Omega \backslash \Sigma \\ u=0 & \text { on } \partial \Omega \cup \Sigma\end{cases}
$$

that means in its weak formulation

$$
\int_{\Omega}|\nabla u|^{p-2} \nabla u \nabla v d x=\int_{\Omega} f v d x \quad \forall v \in W_{0}^{1, p}(\Omega \backslash \Sigma) .
$$

We introduce the family of diffeomorphisms $\varphi_{\varepsilon}(x)=x+\varepsilon X(x)$ where $X$ is a smooth vector field from $\mathbb{R}^{d}$ to $\mathbb{R}^{d}$ supported in $\Omega$. For $\varepsilon$ small enough, $\varphi_{\varepsilon}$ maps $\Omega$ into $\Omega$. Set $\Sigma_{\varepsilon}=\varphi_{\varepsilon}(\Sigma)$ and consider the new state equation in the deformed domain

$$
\begin{cases}-\operatorname{div}\left(\left|\nabla u_{\varepsilon}\right|^{p-2} \nabla u_{\varepsilon}\right)=f & \text { in } \Omega \backslash \Sigma_{\varepsilon} \\ u=0 & \text { on } \partial \Omega \cup \Sigma_{\varepsilon} .\end{cases}
$$

The corresponding functional is

$$
\mathcal{F}\left(\Sigma_{\varepsilon}\right)=\int_{\Omega \backslash \Sigma_{\varepsilon}} F\left(x, u_{\varepsilon}(x), \nabla u_{\varepsilon}(x)\right) d x+\lambda \mathcal{H}^{1}\left(\Sigma_{\varepsilon}\right) .
$$

Notice that for $\varepsilon=0$ equation (8) reduces to (17) and we denote $u_{0}$ simply by $u$. From now on $u$ stands for the unique solution of (7) and $u_{\varepsilon}$ for (8). To differentiate (9) we need to show the differentiability of the function $\varepsilon \mapsto u_{\varepsilon}$ at zero. If we assume $f \in L^{\infty}(\Omega)$ then, by the regularity theory of elliptic equations, $u$ and $u_{\varepsilon}$ are in $W_{0}^{2, p}(\Omega \backslash \Sigma)$ and $W_{0}^{2, p}\left(\Omega \backslash \Sigma_{\varepsilon}\right)$ respectively. Now let us denote by $U_{\varepsilon}=u_{\varepsilon} \circ \varphi_{\varepsilon}$ the transported solution on the fixed domain $\Omega \backslash \Sigma$. Since $U_{\varepsilon} \in W^{2, p}(\Omega \backslash \Sigma)$ (because $\varphi_{\varepsilon}$ is smooth and $u_{\varepsilon} \in W^{2, p}\left(\Omega \backslash \Sigma_{\varepsilon}\right)$ ) the Lemma 4.2 of [7] gives the differentiability of $\varepsilon \mapsto U_{\varepsilon}$ from $\left[0, \varepsilon_{0}\right)$ to $W^{2, p}(\Omega \backslash \Sigma)$ at zero and the differentiability of $\varepsilon \mapsto u_{\varepsilon}$ from $\left[0, \varepsilon_{0}\right)$ to $W_{l o c}^{1, p}(\Omega \backslash \Sigma)$ is obtained via Lemma 2.1 of [8]. Moreover $u^{\prime}=U^{\prime}-\nabla u \cdot X$ in $\Omega \backslash \Sigma$, where $u^{\prime}=\lim _{\varepsilon \rightarrow 0}\left(u_{\varepsilon}-u\right) / \varepsilon$ in the distributional sense. For an open set $D$, taking $v, \varphi \in W^{1, p}(D)$ and $\psi \in C_{c}^{\infty}(D)$, an easy computation of the limit of

$$
\frac{1}{t}\left\langle\Delta_{p}(v+t \varphi)-\Delta_{p}(v), \psi\right\rangle
$$

as $t \rightarrow 0$, gives $\left\langle-\operatorname{div} G_{v}(\nabla \varphi), \psi\right\rangle$ where $\langle\cdot, \cdot\rangle$ is the duality pairing between $\mathcal{D}^{\prime}(D)$ and $C_{c}^{\infty}(D)$, and $G_{v}$ is defined by

$$
G_{v}(Z)=|\nabla v|^{p-2} Z+(p-2)|\nabla v|^{p-4}(\nabla v \cdot Z) \nabla v .
$$

So the differential of $\Delta_{p}$ at a point $v \in W^{1, p}(D)$ is given by

$$
\frac{\partial \Delta_{p}(v)}{\partial v}(\varphi)=-\operatorname{div}\left(G_{v}(\nabla \varphi)\right), \quad \forall \varphi \in W^{1, p}(D) .
$$


At $u$ solution of (7) the differential of $\Delta_{p}$ is linear and continuous from $W^{1, p}(\Omega \backslash \Sigma)$ into $\mathcal{D}^{\prime}(\Omega \backslash \Sigma)$ that is

$$
\frac{\partial \Delta_{p}(u)}{\partial v} \in \mathcal{L}\left(W^{1, p}(\Omega \backslash \Sigma) ; \mathcal{D}^{\prime}(\Omega \backslash \Sigma)\right) .
$$

The fact that $u_{\varepsilon} \in W^{2, p}\left(\Omega \backslash \Sigma_{\varepsilon}\right)$ and solves equation (8), the differentiability of the $p$-Laplacian operator from $W^{1, p}(\Omega)$ to $\mathcal{D}^{\prime}(\Omega)$ and the differentiability of the transported solution $U_{\varepsilon}=u_{\varepsilon} \circ \varphi_{\varepsilon}$ at $\varepsilon=0$ allow to differentiate the equation (8) in the distributional sense at $\varepsilon=0$ (see [8]) and we obtain the equation

$$
-\operatorname{div}\left(G_{u}\left(\nabla u^{\prime}\right)\right)=0 \quad \text { in } \Omega \backslash \Sigma
$$

in the distributional sense. This gives an equation satisfied by $u^{\prime}$ inside $\Omega \backslash \Sigma$. It remains to find the boundary condition of $u^{\prime}$. To this aim, we have to differentiate the boundary condition of $u_{\varepsilon}$. Due to the particular setting of our problem (the boundary operator is the identity and $u_{\varepsilon}=0$ on $\partial \Omega \cup \Sigma_{\varepsilon}$ ), the fact that $u \in W^{2, p}(\Omega \backslash \Sigma)$ and the differentiability of the transported solution $U_{\varepsilon}$ at zero imply the differentiability of the boundary condition (see [8]) and we have

$$
u^{\prime}=0 \text { on } \partial \Omega \quad \text { and } \quad u^{\prime}=-\nabla u \cdot X \text { on } \Sigma .
$$

The fact that $u^{\prime}$ vanishes on the boundary of $\Omega$ is due to the compact support of the vector field $X$ in $\Omega$. So the equation satisfied by $u^{\prime}$ in the distributional sense is

$$
\begin{cases}-\operatorname{div}\left(G_{u}\left(\nabla u^{\prime}\right)\right)=0 & \text { in } \Omega \backslash \Sigma \\ u^{\prime}=0 & \text { on } \partial \Omega \\ u^{\prime}=-\nabla u \cdot X & \text { on } \Sigma .\end{cases}
$$

To complete this part, let us check the differentiability at $\varepsilon=0$ of the cost function. It is well known (see for example [1])that the length functional $\mathcal{H}^{1}\left(\Sigma_{\varepsilon}\right)$ is differentiable at $\varepsilon=0$. The only point to check is concerned with the differentiability of the map $\varepsilon \mapsto \int_{\Omega} F\left(x, u_{\varepsilon}, \nabla u_{\varepsilon}\right) d x$ at $\varepsilon=0$. The smoothness and the growth condition on $F$ imply that the map $v \mapsto F(\cdot, v, \nabla v)$ maps $W^{2, p}(D)$ into $L^{1}(D)$ and is differentiable from $W^{1, p}(D)$ into $\mathcal{D}^{\prime}(D)$ for any open set $D$. Moreover, if $u$ is the solution of equation (7), $F(\cdot, u, \nabla u) \in W^{1,1}(\Omega \backslash \Sigma)$ and the map $\varepsilon \mapsto F\left(\cdot, u_{\varepsilon}, \nabla u_{\varepsilon}\right) \circ \varphi_{\varepsilon}$ is differentiable thanks to the hypothesis on $u_{\varepsilon}$ and $U_{\varepsilon}$. Therefore (see Theorem 3.3 of [8]) we have the differentiability of the cost function at $\varepsilon=0$. Summarizing, by taking the derivative of the functional (8) at $\varepsilon=0$ we get

$$
\left.\frac{\partial}{\partial \varepsilon}\right|_{\varepsilon=0} \mathcal{F}\left(\Sigma_{\varepsilon}\right)=\int_{\Omega}\left(F_{u} u^{\prime}+F_{z} \cdot \nabla u^{\prime}+\operatorname{div}(F X)\right) d x+\left.\lambda \frac{\partial}{\partial \varepsilon}\right|_{\varepsilon=0} \mathcal{H}^{1}\left(\Sigma_{\varepsilon}\right),
$$

where $u^{\prime}$ is the solution of the equation (10). The derivative $\left.\frac{\partial}{\partial \varepsilon}\right|_{\varepsilon=0} \mathcal{H}^{1}\left(\Sigma_{\varepsilon}\right)$ that appears in the above variation, according to Theorem 7.31 of [1], gives

$$
\left.\frac{\partial}{\partial \varepsilon}\right|_{\varepsilon=0} \mathcal{H}^{1}\left(\Sigma_{\varepsilon}\right)=\int_{\Sigma} \operatorname{div}^{\Sigma} X d \mathcal{H}^{1}=-\left\langle H_{\Sigma}, X\right\rangle
$$


As $\Sigma$ is countably $\mathcal{H}^{1}$-rectifiable, $\operatorname{div}^{\Sigma}$ is the projection of the divergence to the approximate tangent line of $\Sigma$ at $\mathcal{H}^{1}$-a.e point of $\Sigma$.

Unfortunately, the quantity $\int_{\Omega}\left(F_{u} u^{\prime}+F_{z} \cdot \nabla u^{\prime}\right) d x$ is not easily exploitable. To overcome this problem we introduce the adjoint state equation

$$
\begin{cases}-\operatorname{div}\left(G_{u}(\nabla q)\right)=F_{u}-\operatorname{div}\left(F_{z}\right) & \text { in } \Omega \backslash \Sigma \\ q=0 & \text { on } \partial \Omega \cup \Sigma\end{cases}
$$

which has to be understood in the distributional sense

$$
\int_{\Omega}\left(F_{u}-\operatorname{div}\left(F_{z}\right)\right) v d x+\int_{\Omega} \operatorname{div}\left(G_{u}(\nabla q)\right) v d x=0 \quad \forall v \in C_{c}^{\infty}(\Omega \backslash \Sigma) .
$$

We are not interested in the regularity of the functions $u$ and $q$ in the whole domain $\Omega$ but only near the optimal set $\Sigma$. The functions $u^{\prime}$ and $q$ are both in $W^{1, p}(\Omega \backslash \Sigma)$. In the variational formulation of the equation (11) if we take $u^{\prime}$ as a test function, we have

$$
\int_{\Omega}\left(F_{u}-\operatorname{div}\left(F_{z}\right)\right) u^{\prime} d x+\int_{\Omega} \operatorname{div}\left(G_{u}(\nabla q)\right) u^{\prime} d x=0 .
$$

Let $\Omega^{+}$and $\Omega^{-}$be two sets such that $\Omega=\Omega^{+} \cup \Omega^{-}$and $\Sigma \subset \partial \Omega^{+} \cap \partial \Omega^{-}$. The sets $\Omega^{+}$and $\Omega^{-}$may be obtained by connecting $\Sigma$ to the boundary of $\Omega$ by pieces of smooth curves. The assumption made on $\Sigma, \partial \Omega$ and $f$ provide sufficient regularity for $u, u^{\prime}, q$ so that the Green formula can be applied to (12). For the sequel, we use the following notation: $\nabla u^{+}$stands for the trace on $\Sigma$ of $\nabla u$ restricted to $\Omega^{+}, \frac{\partial u^{+}}{\partial \nu}$ for the trace of the respective normal derivative, $F_{z}^{+} \cdot \nu=$ $F_{z}\left(x, 0, \nabla u^{+}\right) \cdot \nu$. Similarly $\nabla u^{-}$stands for the trace on $\Sigma$ of $\nabla u$ restricted to $\Omega^{-}$, $\frac{\partial u^{-}}{\partial \nu}$ for the trace of the respective normal derivative, $F_{z}^{-} \cdot \nu=F_{z}\left(x, 0, \nabla u^{-}\right) \cdot \nu$. Recall also that

$$
\nabla u^{ \pm}=\frac{\partial u^{ \pm}}{\partial \nu} \nu
$$

because $u^{ \pm}=u=0$ on $\Sigma$ (i.e the tangential derivative of $u^{ \pm}$over $\Sigma$ vanishes). Let us compute separately the terms of the equation (12). Starting by the first part, we use the regularity of the boundary of $\Omega^{+}$to perform the integration by parts:

$$
A^{+}=\int_{\Omega^{+}}\left(F_{u} u^{\prime}-\operatorname{div}\left(F_{z}\right) u^{\prime}\right) d x=\int_{\Omega^{+}}\left(F_{u} u^{\prime}+F_{z} \cdot \nabla u^{\prime}\right) d x-\int_{\partial \Omega^{+}} u^{\prime} F_{z} \cdot \nu d \mathcal{H}^{1},
$$

where $\nu$ is the outer normal of $\Omega^{+}$. It is easy to observe that $u^{\prime}=0$ on $\partial \Omega \cap \partial \Omega^{+}$ and $\partial \Omega^{+}=\Sigma \cup\left(\partial \Omega^{+} \backslash(\partial \Omega \cup \Sigma)\right) \cup\left(\partial \Omega \cap \partial \Omega^{+}\right)$so

$A^{+}=\int_{\Omega^{+}}\left(F_{u} u^{\prime}+F_{z} \cdot \nabla u^{\prime}\right) d x+\int_{\Sigma} \frac{\partial u^{+}}{\partial \nu}\left(F_{z}^{+} \cdot \nu\right) X \nu d \mathcal{H}^{1}-\int_{\partial \Omega^{+} \backslash(\partial \Omega \cup \Sigma)} u^{\prime} F_{z} \cdot \nu d \mathcal{H}^{1}$. 
Similarly, taking into account the fact that the outer normal of $\Omega^{-}$restricted to $\Omega^{+} \cap \Omega^{-}$is $-\nu$, one gets

$$
A^{-}=\int_{\Omega^{-}}\left(F_{u} u^{\prime}+F_{z} \cdot \nabla u^{\prime}\right) d x-\int_{\Sigma} \frac{\partial u^{-}}{\partial \nu}\left(F_{z}^{-} \cdot \nu\right) X \nu d \mathcal{H}^{1}+\int_{\partial \Omega^{-} \backslash(\partial \Omega \cup \Sigma)} u^{\prime} F_{z} \cdot \nu d \mathcal{H}^{1}
$$

Combining the previous relations and using the fact that the two sets $\partial \Omega^{+} \backslash(\partial \Omega \cup$ $\Sigma)$ and $\partial \Omega^{-} \backslash(\partial \Omega \cup \Sigma)$ coincide, gives $\left(A=A^{+}+A^{-}\right)$

$$
A=\int_{\Omega}\left(F_{u} u^{\prime}+F_{z} \cdot \nabla u^{\prime}\right) d x+\int_{\Sigma}\left(\frac{\partial u^{+}}{\partial \nu} F_{z}^{+} \cdot \nu-\frac{\partial u^{-}}{\partial \nu} F_{z}^{-} \cdot \nu\right) X \nu d \mathcal{H}^{1} .
$$

For the second term, the integration by parts leads to

$$
\begin{aligned}
B^{+} & =\int_{\Omega^{+}} \operatorname{div} G_{u}(\nabla q) u^{\prime} d x \\
& =-\int_{\Omega^{+}} G_{u}(\nabla q) \cdot \nabla u^{\prime} d x+\int_{\partial \Omega^{+}} u^{\prime} G_{u}(\nabla q) \cdot \nu d \mathcal{H}^{1}
\end{aligned}
$$

where $\nu$ is the outer normal of $\Omega^{+}$as in the previous case. It is easily seen that

$$
\begin{aligned}
B^{+}= & -\int_{\Omega^{+}} G_{u}(\nabla q) \cdot \nabla u^{\prime} d x-\int_{\Sigma} \frac{\partial u^{+}}{\partial \nu}\left(G_{u}(\nabla q)^{+} \cdot \nu\right) X \nu d \mathcal{H}^{1} \\
& +\int_{\partial \Omega^{+} \backslash(\partial \Omega \cup \Sigma)} u^{\prime} G_{u}(\nabla q) \cdot \nu d \mathcal{H}^{1} .
\end{aligned}
$$

Similarly, we have

$$
\begin{aligned}
B^{-}= & -\int_{\Omega^{-}} G_{u}(\nabla q) \cdot \nabla u^{\prime} d x+\int_{\Sigma} \frac{\partial u^{-}}{\partial \nu}\left(G_{u}(\nabla q)^{-} \cdot \nu\right) X \nu d \mathcal{H}^{1} \\
& -\int_{\partial \Omega^{-} \backslash(\partial \Omega \cup \Sigma)} u^{\prime} G_{u}(\nabla q) \cdot \nu d \mathcal{H}^{1} .
\end{aligned}
$$

Therefore, summing up one obtains $\left(B=B^{+}+B^{-}\right)$

$$
\begin{aligned}
B= & -\int_{\Omega} G_{u}(\nabla q) \cdot \nabla u^{\prime} d x \\
& +\int_{\Sigma}\left(\frac{\partial u^{-}}{\partial \nu} G_{u}(\nabla q)^{-} \cdot \nu-\frac{\partial u^{+}}{\partial \nu} G_{u}(\nabla q)^{+} \cdot \nu\right) X \nu d \mathcal{H}^{1} .
\end{aligned}
$$

By the linearity of the function $G_{u}$, we get

$$
\int_{\Omega} G_{u}(\nabla q) \cdot \nabla u^{\prime} d x=\int_{\Omega} G_{u}\left(\nabla u^{\prime}\right) \cdot \nabla q d x
$$

but, by integration by parts allowed by regularity of $\Omega$ and $\Sigma$, it follows that

$$
\int_{\Omega} G_{u}\left(\nabla u^{\prime}\right) \cdot \nabla q d x=-\int_{\Omega} \operatorname{div}\left(G_{u}\left(\nabla u^{\prime}\right)\right) q d x+\int_{\partial \Omega \cup \Sigma} q G_{u}\left(\nabla u^{\prime}\right) \cdot \nu d \mathcal{H}^{1}=0
$$


because $u^{\prime}$ is the weak solution of equation (10) and $q$ vanishes on $\partial \Omega \cup \Sigma$. Finally we obtain

$$
\begin{aligned}
\int_{\Omega}\left(F_{u} u^{\prime}+F_{z} \cdot \nabla u^{\prime}\right) d x= & -\int_{\Sigma}\left(\frac{\partial u^{+}}{\partial \nu} F_{z}^{+} \cdot \nu-\frac{\partial u^{-}}{\partial \nu} F_{z}^{-} \cdot \nu\right. \\
& \left.+\frac{\partial u^{-}}{\partial n} G_{u}(\nabla q)^{-} \cdot \nu-\frac{\partial u^{+}}{\partial \nu} G_{u}(\nabla q)^{+} \cdot \nu\right) X \nu d \mathcal{H}^{1} .
\end{aligned}
$$

To compute the term $\int_{\Omega} \operatorname{div}(F X) d x$, we use the divergence theorem thank to the reguality of $\Omega$. Then

$$
\int_{\Omega} \operatorname{div}(F X) d x=\int_{\partial \Omega} F X \nu d \mathcal{H}^{d-1}=0
$$

since $X$ is supported in $\Omega$. It follows that

$$
\begin{aligned}
\left.\frac{\partial}{\partial \varepsilon}\right|_{\varepsilon=0} \mathcal{F}\left(\Sigma_{\varepsilon}\right) & =-\lambda\left\langle H_{\Sigma}, X\right\rangle-\int_{\Sigma}\left(\frac{\partial u^{+}}{\partial \nu} F_{z}^{+} \cdot \nu-\frac{\partial u^{-}}{\partial \nu} F_{z}^{-} \cdot \nu\right) X \nu d \mathcal{H}^{1} \\
& -\int_{\Sigma}\left(\frac{\partial u^{-}}{\partial \nu} G_{u}(\nabla q)^{-} \cdot \nu-\frac{\partial u^{+}}{\partial \nu} G_{u}(\nabla q)^{+} \cdot \nu\right) X \nu d \mathcal{H}^{1}
\end{aligned}
$$

but, by simple computation, we have

$$
\begin{aligned}
G_{u}(\nabla q)^{+} \cdot \nu & =\left|\nabla u^{+}\right|^{p-2} \nabla q^{+} \cdot \nu+(p-2)\left|\nabla u^{+}\right|^{p-4}\left(\nabla u^{+} \cdot \nabla q^{+}\right) \nabla u^{+} \cdot \nu \\
& =\left|\frac{\partial u^{+}}{\partial \nu}\right|^{p-2} \frac{\partial q^{+}}{\partial \nu}+(p-2)\left|\frac{\partial u^{+}}{\partial \nu}\right|^{p-4}\left(\frac{\partial u^{+}}{\partial \nu} \frac{\partial q^{+}}{\partial \nu}\right) \frac{\partial u^{+}}{\partial \nu} \\
& =(p-1)\left|\frac{\partial u^{+}}{\partial \nu}\right|^{p-2} \frac{\partial q^{+}}{\partial \nu}
\end{aligned}
$$

and also similarly

$$
G_{u}(\nabla q)^{-} \cdot \nu=(p-1)\left|\frac{\partial u^{-}}{\partial \nu}\right|^{p-2} \frac{\partial q^{-}}{\partial \nu} .
$$

Combining all the computations together we get

$$
\begin{aligned}
\left.\frac{\partial}{\partial \varepsilon}\right|_{\varepsilon=0} & \mathcal{F}\left(\Sigma_{\varepsilon}\right)=-\lambda\left\langle H_{\Sigma}, X\right\rangle \\
& -\int_{\Sigma}\left(\frac{\partial u^{+}}{\partial \nu} F_{z}^{+} \cdot \nu-\frac{\partial u^{-}}{\partial \nu} F_{z}^{-} \cdot \nu\right) X \nu d \mathcal{H}^{1} \\
& -(p-1) \int_{\Sigma}\left(\left|\frac{\partial u^{-}}{\partial \nu}\right|^{p-2} \frac{\partial u^{-}}{\partial \nu} \frac{\partial q^{-}}{\partial \nu}-\left|\frac{\partial u^{+}}{\partial \nu}\right|^{p-2} \frac{\partial u^{+}}{\partial \nu} \frac{\partial q^{+}}{\partial \nu}\right) X \nu d \mathcal{H}^{1}
\end{aligned}
$$

This equality holds for every vector field $X$, then we derive the following necessary condition of optimality:

$$
\begin{aligned}
& -\lambda\left\langle H_{\Sigma}, \nu\right\rangle-\left(\frac{\partial u^{+}}{\partial \nu} F_{z}^{+} \cdot \nu-\frac{\partial u^{-}}{\partial \nu} F_{z}^{-} \cdot \nu\right)+ \\
& -(p-1)\left(\left|\frac{\partial u^{-}}{\partial \nu}\right|^{p-2} \frac{\partial u^{-}}{\partial \nu} \frac{\partial q^{-}}{\partial \nu}-\left|\frac{\partial u^{+}}{\partial \nu}\right|^{p-2} \frac{\partial u^{+}}{\partial \nu} \frac{\partial q^{+}}{\partial \nu}\right)=0 .
\end{aligned}
$$


We can rewrite this necessary condition of optimality as

$$
\lambda\left\langle H_{\Sigma}, \nu\right\rangle+\left(\frac{\partial u}{\partial \nu} F_{z} \cdot \nu-(p-1)\left|\frac{\partial u}{\partial \nu}\right|^{p-2} \frac{\partial u}{\partial \nu} \frac{\partial q}{\partial \nu}\right)^{ \pm}=0,
$$

where the notation $(a)^{ \pm}$stands for $a^{+}-a^{-}$.

We have proved the following result.

Theorem 4 Let $\Sigma$ be an optimal set in the minimization problem (2) and $u$ the corresponding solution of the state equation. Assume $d=2$, then $u$ satisfies the necessary condition of optimality:

$$
\lambda\left\langle H_{\Sigma}, \nu\right\rangle+\left(\frac{\partial u}{\partial \nu} F_{z} \cdot \nu-(p-1)\left|\frac{\partial u}{\partial \nu}\right|^{p-2} \frac{\partial u}{\partial \nu} \frac{\partial q}{\partial \nu}\right)^{ \pm}=0,
$$

where $\nu$ is the unit normal vector of $\Sigma, H_{\Sigma}$ the generalized mean curvature of $\Sigma$ and $q$ the solution of the adjoint state equation (11).

\section{Case of points in $\mathbb{R}^{d}, d>1$}

In the case of points, some extra difficulties arise because for an equation like (10) the gradient and the normal are not defined on points. The equation (10) is not the crucial point since we are moving an optimal point $x_{0}$ in the direction of the vector field $X$, the boundary condition of (10) may be writen in the general form as $u^{\prime}\left(x_{0}\right)=\frac{\partial u}{\partial X}\left(x_{0}\right)$ if $x_{0}$ is an optimal point. The main difficulty is that in the computation we need an integration by part and since the co-dimention of the point in $\mathbb{R}^{d}(d>1)$ is greater than 1 , there is a lack of an integration by part formulas. The strategy is to study the configurations which are close to the optimal one and obtain the optimal configuration as a limit. We consider, for $r$ small and positive real number, the set $\Sigma_{r}=\psi\left(\overline{B_{r}\left(x_{0}\right)}\right)$ where $B_{r}\left(x_{0}\right)$ is the ball centered at the point $x_{0}$ with radius $r$ and $\psi$ is a smooth diffeomorphism from $\Omega$ to $\Omega$ such that $\psi\left(x_{0}\right)=x_{0}$. The associated state equation is

$$
\begin{cases}-\operatorname{div}\left(|\nabla u|^{p-2} \nabla u\right)=f & \text { in } \Omega \backslash \Sigma_{r} \\ u=0 & \text { on } \partial \Omega \cup \Sigma_{r}\end{cases}
$$

For the functional, we consider

$$
\mathcal{F}\left(\Sigma_{r}\right)=\frac{1}{r^{d-1}} \int_{\Omega \backslash \Sigma_{r}} F(x, u(x), \nabla u(x)) d x+\frac{\lambda}{r^{d-1}} \mathcal{H}^{d-1}\left(\partial \Sigma_{r}\right) .
$$

The factor $\frac{1}{r^{d-1}}$ is in order to avoid the functional to degenerate to the trivial limit functional which vanishes everywhere. For $r$ small, $\mathcal{H}^{d-1}\left(\partial \Sigma_{r}\right) \approx C r^{d-1}$ where $C$ is independent of $r$ and as $r \rightarrow 0$ the solution of the equation (13) converges strongly in $W_{0}^{1, p}(\Omega)$ to the solution of the same equation defined on $\Omega \backslash\left\{x_{0}\right\}$. If 
we denote by $u^{r}$ the solution of the equation (13) for $r>0$ and by $u$ the solution of same equation for $r=0$ (solution on $\Omega \backslash\left\{x_{0}\right\}$ ) we may assume pointwise convergence of $u^{r}$ and $\nabla u^{r}$ to $u$ and $\nabla u$. Thank to the smoothness of $F$ we have also the pointwise convergence of $F\left(\cdot, u^{r}, \nabla u^{r}\right)$ to $F(\cdot, u, \nabla u)$ as $r \rightarrow 0$. This gives heuristically the scaling factor $r^{1-d}$. The same idea for the case of closed connected sets in the next section. To simplify the notation in the formulas, we will denote the solution of the equation (13) by $u$ instead of $u^{r}$. Proceeding as above, that is transforming the domain by $\varphi_{\varepsilon}$, finding the new state equation and the new functional, and taking the derivative of the functional at $\varepsilon=0$, one gets

$$
\left.\frac{\partial}{\partial \varepsilon}\right|_{\varepsilon=0} \mathcal{F}\left(\left(\Sigma_{r}\right)_{\varepsilon}\right)=\frac{1}{r^{d-1}} \int_{\Omega \backslash \Sigma_{r}}\left(F_{u} u^{\prime}+F_{z} \cdot \nabla u^{\prime}+\operatorname{div}(F X)\right) d x-\lambda \frac{1}{r^{d-1}}\left\langle H_{\partial \Sigma_{r}}, X\right\rangle
$$

where $u^{\prime}$ is the solution of the equation

$$
\begin{cases}-\operatorname{div}\left(G_{u}\left(\nabla u^{\prime}\right)\right)=0 & \text { in } \Omega \backslash \Sigma_{r} \\ u^{\prime}=0 & \text { on } \partial \Omega \\ u^{\prime}=-\nabla u \cdot X & \text { on } \partial \Sigma_{r}\end{cases}
$$

$G_{u}\left(\nabla u^{\prime}\right)$ is as before and $H_{\partial \Sigma_{r}}$ is the generalized mean curvature of $\Sigma_{r}$. Using the fact that $x_{0}$ is optimal and $r$ is small enough (we are in a small neighborhood of the optimal point), we obtain $\left.\frac{\partial}{\partial \varepsilon}\right|_{\varepsilon=0} \mathcal{F}\left(\left(\Sigma_{r}\right)_{\varepsilon}\right)=o(1)$.

To overcome the problem of $\nabla u^{\prime}$ as in the previous case, we introduce the adjoint state equation

$$
\begin{cases}-\operatorname{div}\left(G_{u}(\nabla q)\right)=F_{u}-\operatorname{div}\left(F_{z}\right) & \text { in } \Omega \backslash \Sigma_{r} \\ q=0 & \text { on } \partial \Omega \cup \partial \Sigma_{r}\end{cases}
$$

which has to be understood in the distributional sense

$$
\int_{\Omega \backslash \Sigma_{r}}\left(F_{u} v-\operatorname{div}\left(F_{z}\right) v\right) d x+\int_{\Omega \backslash \Sigma_{r}} \operatorname{div}\left(G_{u}(\nabla q)\right) v d x=0 \quad \forall v \in C_{c}^{\infty}\left(\Omega \backslash \Sigma_{r}\right) .
$$

In particular

$$
\int_{\Omega \backslash \Sigma_{r}}\left(F_{u} u^{\prime}-\operatorname{div}\left(F_{z}\right) u^{\prime}\right) d x+\int_{\Omega \backslash \Sigma_{r}} \operatorname{div}\left(G_{u}(\nabla q)\right) u^{\prime} d x=0 .
$$

By integration by parts, the first term of the equation yields

$$
\int_{\Omega \backslash \Sigma_{r}}\left(F_{u} u^{\prime}-\operatorname{div}\left(F_{z}\right) u^{\prime}\right) d x=\int_{\Omega \backslash \Sigma_{r}}\left(F_{u} u^{\prime}+F_{z} \cdot \nabla u^{\prime}\right) d x-\int_{\partial \Sigma_{r}} u^{\prime} F_{z} \cdot \nu_{r} d \mathcal{H}^{d-1}
$$

where $\nu_{r}$ is the inward normal of $\Sigma_{r}$. The computation is quite similar to the case of closed connected subset of $\mathbb{R}^{2}$ and one gets

$$
\int_{\Omega \backslash \Sigma_{r}} \operatorname{div}\left(G_{u}(\nabla q)\right) u^{\prime} d x=-\int_{\partial \Sigma_{r}}\left(\left.(p-1)|| \frac{\partial u}{\partial \nu}\right|^{p-2} \frac{\partial u}{\partial \nu} \frac{\partial q}{\partial \nu}\right) X \nu d \mathcal{H}^{d-1} .
$$


Here $\partial \Sigma_{r}$ plays the role of $\Sigma$ in the two dimensional case. Moreover all the quantities vanish in the interior side of $\Sigma_{r}$ then we are interested only on the exterior side of $\Sigma_{r}$. We obtain

$$
\int_{\Omega \backslash \Sigma_{r}}\left(F_{u} u^{\prime}+F_{z} \cdot \nabla u^{\prime}\right) d x=-\int_{\partial \Sigma_{r}}\left(\frac{\partial u}{\partial \nu} F_{z} \cdot \nu-(p-1)\left|\frac{\partial u}{\partial \nu}\right|^{p-1} \frac{\partial u}{\partial \nu} \frac{\partial q}{\partial \nu}\right) X \nu d \mathcal{H}^{d-1} .
$$

Using the above calculation, one can rewrite the derivative of the functional as

$$
\begin{aligned}
\left.\frac{\partial}{\partial \varepsilon}\right|_{\varepsilon=0} \mathcal{F}\left(\left(\Sigma_{r}\right)_{\varepsilon}\right)= & -\frac{\lambda}{r^{d-1}}\left\langle H_{\partial \Sigma_{r}}, X\right\rangle \\
& -\frac{\lambda}{r^{d-1}} \int_{\partial \Sigma_{r}}\left(\frac{\partial u}{\partial \nu} F_{z} \cdot \nu-(p-1)\left|\frac{\partial u}{\partial \nu}\right|^{p-2} \frac{\partial u}{\partial \nu} \frac{\partial q}{\partial \nu}\right) X \nu d \mathcal{H}^{d-1} .
\end{aligned}
$$

By a change of variables of type $x=\psi(r, \theta), \theta \in S^{d-1}$ we get

$$
\begin{gathered}
-\int_{S^{d-1}} \int_{0}^{r}\left(\frac{\partial u}{\partial \nu} F_{z} \cdot \nu-(p-1)\left|\frac{\partial u}{\partial \nu}\right|^{p-2} \frac{\partial u}{\partial \nu} \frac{\partial q}{\partial \nu}\right) X \nu J(\theta) d r d \theta-\frac{\lambda}{r^{d-1}}\left\langle H_{\partial \Sigma_{r}}, X\right\rangle \\
=o(1) .
\end{gathered}
$$

In this notation all the terms of the integrand are evaluated at $\psi(r, \theta)$ and $J(\theta)$ is the Jacobian determinant of the function: $\theta \mapsto \psi(\theta)$. It remains to study the limit as $r$ tends to 0 . We do it in the particular way by letting $\psi(r, \theta)$ go to $x_{0}$ in a fixed direction as $r$ goes to 0 . To express the dependence of the limit on the direction $\psi(\theta)$, we use the following notation: $\nu(\psi(r, \theta)) \rightarrow \nu(\psi(\theta))$ as $r \rightarrow 0$; the same notation will be also used for other functions in the integrand. This gives:

$$
\int_{S^{d-1}}\left(\frac{\partial u}{\partial \nu} F_{z} \cdot \nu-(p-1)\left|\frac{\partial u}{\partial \nu}\right|^{p-2} \frac{\partial u}{\partial \nu} \frac{\partial q}{\partial \nu}\right) X \nu J d \theta=0
$$

All the terms in the integrand are evaluated at $\psi(\theta)$. The quantity $r^{1-d}\left\langle H_{\partial \Sigma_{r}}, X\right\rangle$ goes to zero as $r$ goes to zero. In fact if we set $\mu_{r}=r^{1-d} \mathcal{H}^{d-1}\left\llcorner\partial \Sigma_{r}\right.$ this measure belongs to $\mathcal{M}_{B C}$ and weakly converges to the Dirac mass $\omega_{d-1} \delta_{x_{0}}$ concentrated at $x_{0}$. Since $\operatorname{dim} \operatorname{Tan}\left(\mu_{r}\right)=d-1$ for all $r>0$ it follows that $\left(\operatorname{dim} \operatorname{Tan}\left(\mu_{r}\right)\right) \mu_{r}=(d-$ 1) $\mu_{r}$ weakly converges to $(d-1) \omega_{d-1} \delta_{x_{0}}$. Therefore since $\operatorname{dim} \operatorname{Tan}\left(\delta_{0}\right)=0<d-1$ we may apply Theorem 3 with $g$ the constant function $d-1$ to have the weak convergence of the mean curvature $H_{\mu_{r}}$ to the mean curvature $H_{\delta_{x_{0}}}$ which is identically zero. As a consequence the generalized mean curvature $H_{\partial \Sigma_{r}}$ of $\Sigma_{r}$ weakly converges to the generalized mean curvature $H_{\delta_{x_{0}}}$ of the point $x_{0}$. Since $\partial \Sigma$ is smooth $H_{\partial \Sigma_{r}}$ coincides with the classical mean curvature but to avoid confusion with mean curvature of measure in this paper, we keep the terminology of general mean curvature. The equality in (15) holds for every $X \in C_{c}^{\infty}(\Omega)$ and every $\psi$ diffeomorphism. Again it holds true for $X$ constant in the neighborhood of the optimal point and for all $\psi$ diffeomorphism satisfying the condition

$$
\int_{S^{d-1}} \nu(\psi(\theta)) J(\theta) d \theta=0
$$


This allows us to write

$$
\frac{\partial u}{\partial \nu} F_{z} \cdot \nu-(p-1)\left|\frac{\partial u}{\partial \nu}\right|^{p-2} \frac{\partial u}{\partial \nu} \frac{\partial q}{\partial \nu}=\text { const }
$$

This expression which is evaluated at $\psi(\theta)$ is constant for all $\psi$ and $\theta \in S^{d-1}$. This means that it is constant in any direction. Then we have the necessary condition of optimality:

$$
\frac{\partial u}{\partial \nu} F_{z} \cdot \nu-(p-1)\left|\frac{\partial u}{\partial \nu}\right|^{p-2} \frac{\partial u}{\partial \nu} \frac{\partial q}{\partial \nu}=\text { const }
$$

Let us consider a particular case of this problem. We assume $d=2$ and $F=f(x) u$ where $u$ is the solution of the $p$-Laplacian equation. To express the dependence of $u$ on $p$ we denote it by $u_{p}$ instead of $u$ and the same rule for $q$. Since $p>2$ we want to study the limit as $p \rightarrow 2^{+}$of the problem. The sequence $u_{p}$ are bounded in $H_{0}^{1}(\Omega \backslash \Sigma)$ then up to extracting a subsequence, it converges weakly to some function $u$. It is easy to see that $u$ coincides with the solution of the classical Laplacian that is the solution of equation (11) when $p=2$. From the adjoint state equation (14) we may deduce also that the limit of $q_{p}$ as $p \rightarrow 2^{+}$coincides with the solution of equation (11) for $p=2$. We may then rewrite the necessary condition of optimality in the form:

$$
\left|\frac{\partial u}{\partial \nu}\right|=\text { const }
$$

The result proved is summarized below.

Theorem 5 Let $\Sigma$ be an optimal set in the minimization problem (3), where $d>1$ and $u$ is the solution of the corresponding state equation. Then $u$ satisfies the necessary condition of optimality:

$$
\frac{\partial u}{\partial \nu} F_{z} \cdot \nu-(p-1)\left|\frac{\partial u}{\partial \nu}\right|^{p-2} \frac{\partial u}{\partial \nu} \frac{\partial q}{\partial \nu}=\text { const }
$$

where $\nu$ and $q$ are respectively the limit as $r \rightarrow 0$ in a given direction of the unit normal vector of $\Sigma_{r}$ and the solution of the adjoint state equation (15).

The case of points in $\mathbb{R}$ is similar to the case of closed connected subsets in $\mathbb{R}$.

\section{Case of closed connected subsets in $\mathbb{R}^{d}$ with $d>$ 2}

Here the strategy is the same. Let $\Sigma$ be the optimal configuration. We study the configuration which is close to the optimal one and pass to the limit. As in the 
case of points, we consider a tube $\Sigma_{r}=\left\{x \in \mathbb{R}^{n}: d(x, \Sigma) \leq r\right\}$. The associated state equation is

$$
\begin{cases}-\operatorname{div}\left(|\nabla u|^{p-2} \nabla u\right)=f & \text { in } \Omega \backslash \Sigma_{r} \\ u=0 & \text { on } \partial \Omega \cup \Sigma_{r}\end{cases}
$$

The procedure is quite similar to the previous case. The corresponding general functional is

$$
\mathcal{F}\left(\Sigma_{r}\right)=\frac{1}{\mathcal{H}^{d-2}\left(S_{r}^{d-2}\right)} \int_{\Omega \backslash \Sigma_{r}} F(x, u(x), \nabla u(x))+\frac{\lambda}{\mathcal{H}^{d-2}\left(S_{r}^{d-2}\right)} \mathcal{H}^{d-1}\left(\partial \Sigma_{r}\right),
$$

where $S_{r}^{d-2}$ is a $(d-2)$-dimensional sphere of radius $r$ and centered on points of $\Sigma$. From the previous computation, we deduce the derivative of the functional:

$$
\begin{aligned}
\left.\frac{\partial}{\partial \varepsilon}\right|_{\varepsilon=0} & \mathcal{F}\left(\left(\Sigma_{r}\right)_{\varepsilon}\right)=-\frac{\lambda}{\mathcal{H}^{d-2}\left(S_{r}^{d-2}\right)}\left\langle H_{\partial \Sigma_{r}}, X\right\rangle \\
& -\frac{1}{\mathcal{H}^{d-2}\left(S_{r}^{d-2}\right)} \int_{\partial \Sigma_{r}}\left(\frac{\partial u}{\partial \nu} F_{z} \cdot \nu-(p-1)\left|\frac{\partial u}{\partial \nu}\right|^{p-2} \frac{\partial u}{\partial \nu} \frac{\partial q}{\partial \nu}\right) X \nu d \mathcal{H}^{d-1},
\end{aligned}
$$

where $H_{\partial \Sigma_{r}}$ is the generalized mean curvature of $\partial \Sigma_{r}$. Remark that all the equations are the same as in the case of points in $\mathbb{R}^{d}$. To pass to the limit, we argue as in the case of points in $\mathbb{R}^{d}$. First we disintegrate the measure $\mathcal{H}^{d-1}$ and get

$$
\begin{aligned}
& \left.\frac{\partial}{\partial \varepsilon}\right|_{\varepsilon=0} \mathcal{F}\left(\left(\Sigma_{r}\right)_{\varepsilon}\right)=-\frac{\lambda}{\left|\Sigma_{r}\right|}\left\langle H_{\partial \Sigma_{r}}, X\right\rangle \\
& -\frac{1}{\mathcal{H}^{d-2}\left(S_{r}^{d-2}\right)} \int_{\Sigma} \int_{S_{r}^{d-2}}\left(\frac{\partial u}{\partial \nu} F_{z} \cdot \nu-(p-1)\left|\frac{\partial u}{\partial \nu}\right|^{p-2} \frac{\partial u}{\partial \nu} \frac{\partial q}{\partial \nu}\right) X \nu d \mathcal{H}^{d-2} d \mathcal{H}^{1}
\end{aligned}
$$

The measure $\mathcal{H}^{d-2}\left(S_{r}^{d-2}\right)^{-1} \mathcal{H}^{d-2}\left\llcorner S_{r}^{d-2}\right.$ converges weakly to $\delta_{x}$ where $x$ is the center of the sphere $S_{r}^{d-2}$. Due to the hypothesis made on data of the problem the measure

$$
\left(\frac{\partial u}{\partial \nu} F_{z} \cdot \nu-(p-1)\left|\frac{\partial u}{\partial \nu}\right|^{p-2} \frac{\partial u}{\partial \nu} \frac{\partial q}{\partial \nu}\right) \nu \mathcal{H}^{d-2}\left(S_{r}^{d-2}\right)^{-1} \mathcal{H}^{d-2}\left\llcorner S_{r}^{d-2}\right.
$$

weakly converges to the measure

$$
\left(\frac{\partial u}{\partial \nu} F_{z} \cdot \nu-(p-1)\left|\frac{\partial u}{\partial \nu}\right|^{p-2} \frac{\partial u}{\partial \nu} \frac{\partial q}{\partial \nu}\right) \nu \delta_{x}
$$

for $\mathcal{H}^{1}$ a.e. $x \in \Sigma$. The limit here is computed in a fixed direction $\theta \in S^{d-2}$. For the curvature part, notice first that the measure $\mu_{r}=\mathcal{H}^{d-2}\left(S_{r}^{d-2}\right)^{-1} \mathcal{H}^{d}\left\llcorner\partial \Sigma_{r} \in\right.$ $\mathcal{M}_{B C}$ weakly converges to the measure $\mathcal{H}^{1}\left\llcorner\Sigma\right.$ and $\left(\operatorname{dim} \operatorname{Tan}\left(\mu_{r}\right)\right) \mu_{r}=d \mu_{r}$ weakly converges to the measure $d \mathcal{H}^{1}\left\llcorner\Sigma\right.$. The fact that $\operatorname{dim} \operatorname{Tan}^{1}\llcorner\Sigma=1<d$ allows to apply again Theorem 3 to have weak convergence of the mean curvature of $\mu_{r}$ to 
that of $\mathcal{H}^{1}\left\llcorner\Sigma\right.$ and consequently the weak convergence of $H_{\partial \Sigma_{r}}$ to $H_{\Sigma}$. Summarizing all computed results we get

$$
\begin{aligned}
\left.\frac{\partial}{\partial \varepsilon}\right|_{\varepsilon=0} \mathcal{F}\left(\Sigma_{\varepsilon}\right)= & -\lambda\left\langle H_{\Sigma}, X\right\rangle \\
& -\int_{\Sigma}\left(\frac{\partial u}{\partial \nu} F_{z} \cdot \nu-(p-1)\left|\frac{\partial u}{\partial \nu}\right|^{p-2} \frac{\partial u}{\partial \nu} \frac{\partial q}{\partial \nu}\right) X \nu d \mathcal{H}^{1}=0 .
\end{aligned}
$$

This relation being true for every vector field $X$, we get the necessary condition of optimality:

$$
\left(\frac{\partial u}{\partial \nu} F_{z} \cdot \nu-(p-1)\left|\frac{\partial u}{\partial \nu}\right|^{p-2} \frac{\partial u}{\partial \nu} \frac{\partial q}{\partial \nu}\right)+\lambda\left\langle H_{\Sigma}, \nu\right\rangle=0
$$

Theorem 6 Let $\Sigma$ be an optimal set in the minimization problem (2), where $d>2$ and $u$ is the solution of the corresponding state equation. Then $u$ satisfies the necessary condition of optimality:

$$
\left(\frac{\partial u}{\partial \nu} F_{z} \cdot \nu-(p-1)\left|\frac{\partial u}{\partial \nu}\right|^{p-2} \frac{\partial u}{\partial \nu} \frac{\partial q}{\partial \nu}\right)+\lambda\left\langle H_{\Sigma}, \nu\right\rangle=0
$$

where $\nu$ is the unit normal vector of $\Sigma$ in a given direction, $H_{\Sigma}$ the generalized mean curvature of $\Sigma$ and $q$ the limit as $r \rightarrow 0$ of the solution of the adjoint state equation.

Remark that this necessary condition of optimality depends on the direction $\theta \in S^{d-2}$. Those directions are contained in the $d-1$ plane which is orthogonal to the approximate tangent line to $\Sigma$.

Acknowledgments. The work of the second author has been performed during the Ph. D. studies at the Department of Mathematics of University of Pisa. He also wish to thank the ICTP of Trieste for a visiting fellowship. The authors wish to thank the anonimous referees for their thorough review and highly appreciate the comments and suggestions, which significantly contributed to improving the quality of the publication.

\section{References}

[1] L. Ambrosio, N. Fusco, D. Pallara: Functions of Bounded Variation and Free Discontinuity Problems. Oxford Mathematical Monographs, Oxford University Press, Oxford, 2000.

[2] G. Bouchitté, G. Buttazzo, I. Fragala: Mean curvature of a measure and related variational problems. Ann. Scuola Norm. Sup. Pisa Cl. Sci., 25 (1997), 179-196. 
[3] D. Bucur, P. Trebeschi: Shape optimization governed by nonlinear state equations. Proc. Roy. Soc. Edinburgh Sec. A, 128 (1998), 945-963.

[4] G. Buttazzo, E. Mainini, E. Stepanov: Stationary configurations for the average distance functional and related problems. Control Cybernet., 38 (2009), $1107-1130$.

[5] G. Buttazzo, F. Santambrogio: Asymptotical compliance optimization for connected networks. Netw. Heterog. Media, 2 (2007), 761-777.

[6] A. Henrot, M. Pierre: Variation et Optimisation de Formes, une Analyse Géométrique. Springer-Verlag, Berlin (2005).

[7] F. Murat, J. Simon: Sur le contrôle par un domaine gémétrique. Rapport du L.A.N. 189, Univ. Paris VI (1976).

[8] J. Simon: Differentiation with respect to the domain variation in boundary value problems. Numer. Fonct. Anal. and Optimiz., 2 (1980), 649-687.

[9] J. Sokolowski, J.P. Zolésio: Introduction to Shape Optimization, Shape Sensitivity Analysis. Springer Series in Computational Mathematics vol. 16, Springer-Verlag, Berlin (1992).

[10] Hua Su: Positive solutions of two-point boundary value problems for n-order p-Laplacian operator. Int. J. Dyn. Syst. Differ. Equ. (IJDSDE), 1 (2) (2007), 132-138.

[11] V. Šverák: On optimal shape design. J. Math. Pures Appl., 72 (1993), 537551.

Giuseppe Buttazzo: Dipartimento di Matematica, Università di Pisa

Largo B. Pontecorvo 5, 56127 Pisa - ITALY

buttazzo@dm.unipi.it

Al-hassem Nayam: International Centre for Theoretical Physics

Strada Costiera 11, 34151 Trieste - ITALY

anayam@ictp.it 